\title{
Análise da estigmatização no contexto do HIV/AIDS: Concepções de Pessoas que Vivem com HIV/AIDS
}

\section{Analysis of Stigmatization in the Context of HIV/AIDS: Concepts of People Living with HIV/AIDS}

\author{
Luciana Kelly da Silva Fonseca (orcid.org/0000-0001-8832-5261)1 \\ José Victor De Oliveira Santos (orcid.org/0000-0002-6661-2873)2 \\ Ludgleydson Fernandes de Araújo (orcid.org/0000-0003-4486-7565) ${ }^{3}$ \\ Alice Vitória Freire Cordeiro Sampaio (orcid.org/0000-0002-7912-0202) ${ }^{4}$
}

\begin{abstract}
Resumo
A presente pesquisa buscou identificar as concepções acerca do estigma social entre pessoas vivendo com o HIV/AIDS. Participaram 44 soropositivos, com idade entre 23 e 67 anos (M = 41,00; DP = 10,60), que estavam em acompanhamento no centro de testagem e aconselhamento (CTA) da Secretária da Saúde da Prefeitura Municipal de Parnaíba/PI. Utilizou-se entrevista semiestruturada e questionário sociodemográfico para caracterização da amostra. Constatou-se que, apesar de quase três décadas passadas da epidemia e da sua mudança epidemiológica, as pessoas que convivem com HIV/AIDS ainda sofrem e internalizam estigmas, que são construções históricas, culturais e sociais, que giram em torno da doença, assim demonstrando medo do julgamento moral da sociedade. Espera-se que esses dados possam subsidiar futuras intervenções em saúde coletiva que possam contribuir para atenuar as vivências de estigma social no contexto da soropositividade para o HIV/AIDS.
\end{abstract}

Palavras-chave: Estigma social. Preconceito. Pessoas vivendo com HIV/AIDS.

\begin{abstract}
The current research sought to identify the conceptions about the social stigma among people living with HIV/AIDS. Participated 44 HIV-positive individuals, aged between 23 and 67 years $(\mathrm{M}=41,00, \mathrm{SD}=10,60)$, who were being monitored at the testing and counseling center (CTA) of the Secretary of Health of the Municipality of Parnaíba/PI, Brazil. Semi-structured interview and sociodemographic questionnaire were used to characterize the sample. It was found that, despite almost three decades after the epidemic and its epidemiological change, people living with HIV/AIDS still suffer and internalize stigmas, which are historical,

\footnotetext{
${ }^{1}$ Universidade Federal do Piauí, Parnaíba, Brasil: E-mail: 1.kelly_fonseca@ hotmail.com.

${ }^{2}$ Universidade Federal do Piauí, Parnaíba, Brasil. E-mail: victorolintos@ hotmail.com.

${ }^{3}$ Universidade Federal do Piauí, Parnaíba, Brasil. E-mail: ludgleydson@yahoo.com.br.

${ }^{4}$ Faculdade Internacional do Delta, Parnaíba, Brasil. E-mail: alicevitoria@globo.com.
} 
cultural and social constructions, revolving around the disease, as well showing fear of the moral judgment of society. It is expected that these data may support future public health interventions that can contribute to alleviate the experiences of social stigma in the context of HIV/AIDS seropositivity.

Keywords: Social stigma. Prejudice. People living with HIV/AIDS. 


\section{Introdução}

Transcorrido três décadas desde a aparição dos primeiros casos de infecção pelo HIV/AIDS, observa-se essa enfermidade como problema de saúde de ordem mundial (Reis, Melo, Galvão \& Gir, 2014) e uma das doenças que representa um dos sérios problemas de saúde pública, caracterizada como epidemia global (Unaids, 2015). A infecção pelo HIV/AIDS é considerada uma doença crônica, o que implica para os profissionais de saúde desafios que vão além de ações voltadas para a contenção da epidemia. A partir disso, percebese que as ações direcionadas para o tratamento e a disseminação de conhecimento são de suma importância por parte dos responsáveis para que não ocorra discriminação ou propagação do estigma social (Araújo, Teva, Quero, Reyes \& Bermúdez, 2017; Araújo, Teva \& Bermúdez, 2015; Santos, 2014; Teva, Araújo \& Bermúdez, 2018).

Nesse contexto, estima-se que 37.967.000 de pessoas vivem com HIV/AIDS globalmente, 17,1 milhões não sabem que têm o vírus e precisam ser atingidos com serviços de testagem do HIV, e por volta de 22 milhões não têm acesso ao tratamento de HIV/AIDS (Unaids, 2015). No território Brasileiro, 830.000 pessoas vivem com HIV/AIDS (Unaids, 2015). Em nível de estado, o Piauí registrou cerca de 2.164 casos de AIDS entre 2004 e 2013, sendo que a maior taxa de incidência no estado ao longo da série histórica foi observada em 2009, quando foi verificada uma taxa de 912 óbitos (Secretaria de Vigilância em Saúde - SVS, 2011).

$\mathrm{Na}$ década de 1990, surgiram os medicamentos chamados de antirretrovirais (ART), que trouxeram uma melhor qualidade de vida às pessoas vivendo com HIV/AIDS (Araújo, 2014). Esses medicamentos são utilizados para o controle da enfermidade, ajudando a prolongar a vida ativa das pessoas infectadas e diminuindo as chances de ação de doenças oportunistas (Unaids, 2015). Em 2013, mais 2,3 milhões de pessoas passaram a ter acesso a esses medicamentos, o que elevou o número global de indivíduos recebendo ART para cerca de 13 milhões (Associação Brasileira de Estudos Populacionais - ABEP, 2014). Apesar da adesão aos ART, um número estimado de 8 milhões de pessoas morreu de causas relacionadas com a HIV/AIDS em 2010 (Qiao, Li \& Stanton, 2014), desse modo, observa-se a necessidade de pesquisas com escopo de conhecer os fatores psicossociais intrínsecos a essa enfermidade.

O surgimento do HIV/AIDS é visto como um fator social e histórico que trouxe sob sua incumbência uma carga de vulnerabilidade social marcante, bem como alguns preconceitos e dúvidas a respeito dessa doença, ainda desconhecida na época de seu surgimento (Araújo, 2014; Carvalho \& Paes, 2011). Sendo assim, desenvolveu-se a estigmatização social que é até hoje vivida de diferentes formas pelas pessoas que convivem com HIV/AIDS. Esse construto pode ser definido como um modo de desprestígio ou desqualificação de um indivíduo em função de possuir ou ter a possibilidade de presença do HIV/AIDS (Zambedetti, 2014).

Atualmente, estigma social e preconceito são prioridades nas pesquisas de vários órgãos governamentais, tendo em vista 
que, apesar de ser largamente aceito o destaque sobre as consequências destes, faltam dados sobre como lidar de fato com essa problemática (Moreira, Meneses, Andrade \& Araújo, 2010). A estigmatização causa alguns danos mentais e sociais negativos na vida de quem a sofre, na medida em que para o estigmatizado as relações sociais têm papel determinante em sua vida (Durães, 2011).

Por causa desses construtos atrelados a essa doença, essas pessoas têm dificuldade em falar com outros indivíduos sobre o diagnóstico, especialmente com pessoas com as quais possivelmente podem desenvolver algum tipo de relacionamento sexual ou afetivo, com medo de serem rejeitados. Nesse sentido, diversos são os impactos relacionados a essa doença. Consequentemente, a culpa por ter se infectado, a não adesão à medicação, a revelação da identidade social, a revolta e o consumo exagerado de bebida alcoólica podem ser citados como alguns dos aspectos associados a essa doença exprobada (Carvalho \& Paes, 2011). Ademais, a chegada dos medicamentos antirretrovirais ajudou na melhora relevante nas condições de saúde de pessoas infectadas pelo HIV/AIDS, permitindo ganhos no processo de desenvolvimento e na qualidade de vida (Guerra \& Seidl, 2009). Porém, quando existe a descoberta da soropositividade, nasce no individuo uma gama de aflições e perturbações que vão além de questões biológicas, perpassando pelos âmbitos pessoal e social.

Logo, destaca-se a necessidade de estudos que sejam condizentes com $\mathrm{O}$ reconhecimento da repercussão negativa do estigma social e da discriminação relacionado ao
HIV/AIDS. Portanto, a relevância social e acadêmica deste estudo resulta em possibilitar conhecer os aspectos de estigma e discriminação social no contexto da soropositividade para HIV/AIDS. Para isso, esta investigação objetiva identificar as concepções acerca do estigma social e preconceito vivenciado por pessoas vivendo com o HIV/AIDS.

\section{Método}

\section{Tipo da Investigação}

Trata-se de uma pesquisa qualitativa, do tipo não probabilística e com dados transversais.

\section{Lócus da Investigação}

A presente investigação foi realizada no Centro de Testagem e Aconselhamento (CTA), na cidade de Parnaíba/PI, cujo espaço é destinado à vigilância, prevenção e controle das Infecções Sexualmente Transmissíveis, do HIV/AIDS e hepatites virais.

\section{Participantes}

Contou-se com 44 pessoas adultas vivendo com HIV/AIDS (de um total de 58 pessoas com HIV) que estavam em acompanhamento no centro de testagem e aconselhamento (CTA), de ambos os sexos, com idades entre 23 e 67 anos $(M=41,00$; DP= 10,60). Destes, $54,9 \%$ são solteiros, 25,5\% casados e o restante da amostra é composto por divorciados ou separados e viúvos. No que concerne à escolaridade, $41,2 \%$ dos participantes têm ensino fundamental incompleto; 19,6\%, ensino médio completo e $15,7 \%$, ensino médio 
incompleto; analfabetos caracterizam $11,8 \%$ da amostra e ensino fundamental completo, ensino superior completo e ensino superior completo atingiram 11,8\% da amostra. Quanto à orientação sexual, $78,4 \%$ dos participantes são heterossexuais e 11,8\% são homoafetivos e $9,8 \%$ são bissexuais. No que diz respeito à renda mensal, cerca de $41,2 \%$ relataram que recebem menos de um salário mínimo, $11,8 \%$ recebem entre dois e três salários mínimos e 3,9\% recebem entre três e cinco salários mínimos.

\section{Instrumentos}

Os resultados decorrentes são descritos em três partes, construídos a partir das seguintes perguntas estruturadas: Você já viveu algum tipo de preconceito ou estigma por ser uma pessoa vivendo com HIV? Como ocorreu essa vivência de preconceito/estigma?

Utilizou-se, ainda, um questionário sociodemográfico para caracterização da população investigada, contemplando: idade, sexo, estado civil, religiosidade ou espiritualidade, orientação sexual, grau de escolaridade, renda familiar.

\section{Procedimentos Éticos}

Inicialmente, esta investigação foi enviada para apreciação do Comitê de Ética em Pesquisa (CEP) da Universidade Federal do Piauí. Depois da aprovação (CAAE n. 48518815.2.0000.5669), foi mantido contato com o CTA para verificar a disponibilidade da realização da referida pesquisa com os usuários dessa instituição de saúde. Depois disso, foi iniciada a coleta de dados, que foi realizada de forma individual, voluntária e anônima entre as pessoas que vivem com HIV/AIDS por quatro estudantes de iniciação científica que foram previamente treinados. $\mathrm{Na}$ aplicação dos instrumentos, foram explicitados os objetivos do estudo e a obtenção das devidas autorizações de participação se deu com o preenchimento do Termo de Consentimento Livre e Esclarecido, de acordo com o disposto na Resolução n. 510/2016 do Conselho Nacional de Saúde (CNS) para que os participantes pudessem autorizar sua participação na pesquisa e responder aos instrumentos.

É válido salientar que foram garantidos sigilo e confidencialidade das respostas dos participantes e que a desistência poderia ocorrer em qualquer momento. O tempo médio para aplicação dos instrumentos entre os participantes foi de 30 minutos e não houve desistência ou recusa por parte de nenhum participante da pesquisa.

\section{Procedimento de Análises dos Dados}

No tratamento da análise dos dados, utilizou-se o programa de computador Iramuteq - Interface de $\mathrm{R}$ Pour Analyses Multidimensionnelles de Textes et de Questionnaires (Camargo \& Justo, 2013; Nascimento \& Menandro, 2006; Ratinaud, 2009), que é compilado a partir do Software R (R Development Core Team, 2011), que analisou os córpus textuais atribuídos às perguntas respondidas pelos participantes do estudo. Tais córpus foram organizados no editor de texto OpenOffice Writer, que foram 
compostos por 44 Unidades de Contextos Iniciais (UCI), que corresponde ao conteúdo textual elaborado pelo participante.

Foram executadas três tipos de análises para cada córpus: primeiramente, foi realizada a Classificação Hierárquica Descendente (CHD), que compila o conteúdo dos textos baseado na frequência e qui-quadrado $\left(\mathrm{X}^{2}\right)$, em que os conteúdos relevantes das UCI são selecionados e divididos em classes de proximidade semântica, surgindo as Unidades de Contexto Elementar (UCE); logo depois, fez-se uso da nuvem de palavras, que organiza as palavras a partir da frequência de incidência e, por último, foi feita a análise de similitude para documentar a coocorrência entre palavras presentes nas falas dos participantes e então associá-las por grau de semelhança (Ratinaud \& Marchand, 2012).

\section{Resultados e Discussões}

Os resultados das perguntas estruturadas sobre o estigma social entre pessoas que vivem com HIV/AIDS compuseram um córpus textual em que a CHD foi obtida a partir das respostas dos participantes, que se referem às opiniões das pessoas que convivem com o HIV/AIDS acerca das dificuldades e vivências de situações de preconceito, discriminação e estigma no contexto da infecção pelo HIV/AIDS. O córpus foi formado por 44 UCI e, ao ser executada a análise de entrada de dados no Iramuteq, ficou dividido em 29 Unidades de Contexto Elementar (UCE).

Foram executados três tipos de análises para o córpus textual, as quais são complementares e possibilitam a visualização dos dados em três performances diferenciadas, possibilitando uma triangulação de resultados. Inicialmente, foi feita a Classificação Hierárquica Descendente (CHD), que correlaciona o conteúdo dos textos baseado na frequência e qui-quadrado $\left(\mathrm{X}^{2}\right)$, criando classes de Unidades de Contextos Elementares (UCE) com vocábulos semelhantes entre si e ao mesmo tempo diferente das UCE das demais classes, sendo essas classes representadas por meio do dendograma (ver Figura 1), que ilustra as relações entre as classes (Camargo, 2005). As classes foram ainda nomeadas e descritas a partir do conteúdo evidenciado nelas. Em seguida, foi realizada a análise de nuvem de palavras, que agrupa as palavras em função da frequência e exibe o resultado de forma gráfica. E, finalmente, a análise de similitude, que apresenta um desenho indicando a conexão entre as palavras, pelo qual é possível identificar as coocorrências destas.

A CHD, obtida a partir das respostas da pergunta, refere-se à exposição ou não do resultado de HIV reagente e da ocorrência ou não de discriminação/estigma social. Das 28 UCE que surgiram, 22 foram significantes na Classificação Hierárquica Descendente, equivalendo a 78,6\% do total, com 319 palavras distintas, que ocorreram 783 vezes, com uma média de 3,26 em termos de ocorrência. No dendograma, estão expostas as duas classes em que o córpus se dividiu, com o título e a descrição de cada uma delas (ver Figura 1), o número de UCE que a compõe, as variáveis descritivas e as palavras que mais se associam com a classe em questão, considerando o coeficiente do teste de associação $X^{2}$. 

Figura 1. Dendograma da Classificação Hierárquica Descendente da estigmatização entre pessoas que vivem com HIV

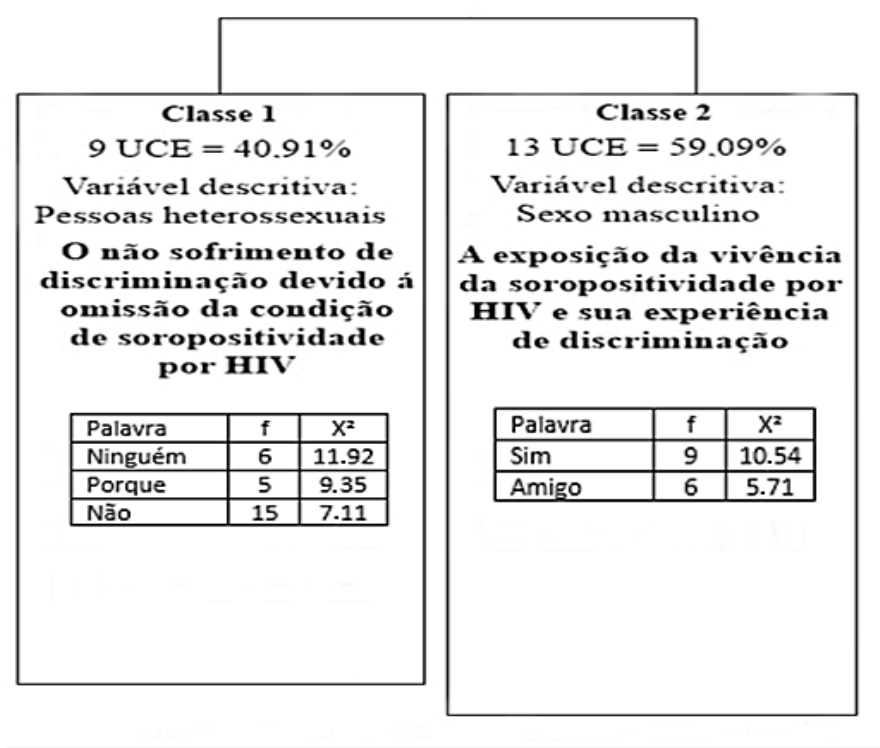

Fonte: Elaborada pelos autores.

A CHD é composta pelas classes 1 e 2 , sendo que a primeira classe diz respeito à "Ausência de sofrimento psicossocial e à omissão da condição de HIV”. Nessa classe, o sofrimento é relatado pelas pessoas que convivem com a doença como omisso, pois elas descrevem que não haviam compartilhado para ninguém por motivos diversos, então não tinham padecido por nenhum constrangimento. No que tange à segunda classe, denominada "Exposição da condição de HIV e as vivências de estigma social", pode-se constatar que as pessoas que vivem com HIV/AIDS demonstraram que já haviam compartilhado sua condição de soropositivo para pessoas próximas e amigos, de modo que sofreram algum tipo de preconceito, xingamento e/ou terror social.

\section{Classe 1: Ausência de Sofrimento Psicossocial e a Omissão da Condição de HIV}

A primeira classe é apresentada de maneira que a condição de soropositividade é ocultada, ou seja, as pessoas relataram que não sofreram discriminação devido à condição de recusa da exposição do diagnóstico, que é representada por $40,91 \%$ das UCE. Tal fato assinala que as pessoas vivendo com HIV/AIDS não compartilham sua condição de saúde e, por isso, não sofrem nenhuma discriminação/estigma da sociedade, como pode ser observado na fala de uma participante cujo grupo etário varia de 47 a 54 anos: "Não, pois eu não conto para as outras pessoas que tenho HIV por ter medo da reação de outras pessoas". Isso assinala a questão de que o estigma ainda hoje é um ator principal na produção e 
perpetuação de poder e de controle em todos os campos sociais, fazendo com que alguns grupos sejam desvalorizados (Parker \& Aggleton, 2001). Essa recusa versa sobre um adiamento da revelação do diagnóstico afetando diversas áreas, como o acesso aos cuidados de saúde e à vida sexual, afetiva, social e educacional (Kourrouski \& Lima, 2009).

Tal fato é salientado por Maldonado, Blanco e Rendón (2016), quando os autores destacam que o estigma atrelado ao HIV/AIDS pode ser de duas formas: externa, que é quando se têm uma percepção negativa da pessoa que convive com HIV/AIDS, e isso faz com que as demais pessoas se afastem de um lugar social; e a segunda forma seria interna, quando as construções externas ao redor da doença são internalizadas pelas pessoas que convivem com a enfermidade e que afetam a sua própria avaliação, tratamento e até mesmo na forma de expressão da doença. Como pode ser verificado na fala de um participante do sexo masculino, grupo etário 55 anos ou mais: "Ainda não, porque tenho vergonha de contar para as outras pessoas".

Destarte, as pessoas que vivem com HIV/AIDS mencionam não haver vantagem em compartilhar sua condição de HIV reagente. Ilustra-se tal questão na fala desta participante, grupo etário entre 31 e 38 anos: "Não, até porque não conto para outras pessoas e faz pouco tempo que fui infectada, não vejo necessidade de contar a ninguém". O construto estigma que ainda permeia a enfermidade pode interferir na rede de apoio social das pessoas soropositivas, causando sofrimento e medo do afastamento das pessoas queridas e, por isso, os infectados acabam escondendo e até mesmo se isolando dos outros para manter $\mathrm{O}$ relacionamento com os amigos e a família (Araújo, 2014; Carvalho \& Paes, 2011).

Pesquisas apontam para o fato de que o contexto da soropositividade para HIV é de vivências e atitudes sociais discriminatórias, de modo que alguns empecilhos contribuem para que o resultado do exame de HIV reagente não seja divulgado, sendo de grande importância a manutenção do sigilo desse resultado para as pessoas que convivem com essa enfermidade (Araújo et al., 2015; Galvão, 2009), fazendo com que as pessoas que são acometidas se camuflem para que não sejam descobertas, o que fica explícito nesta fala de um participante, grupo etário 39 a 46 anos: "Não, sou uma pessoa simples e normal".

\section{Classe 2: Exposição da Condição de HIV e as Vivências de Estigma Social}

No que tange à segunda classe, representada por $59,09 \%$ das UCE, em que são demonstradas as vivências de sofrimento psíquico dos soropositivos ocasionado pelos relatos de preconceitos e estigmas sociais que os entrevistados já sofreram, de familiares e/ou pessoas próximas, resultante do compartilhamento de sua condição de HIV reagente.

Os familiares foram apontados na pesquisa como sendo uma das principais fontes de apoio ou não para os indivíduos que são acometidos pela doença. Em recente estudo, Carvalho e Paes (2011) destacaram que se afastar do contato familiar significa prejuízos 
para as pessoas que são acometidas pelo HIV/AIDS. No entanto, o que foi encontrado é que nem sempre familiares oferecem alento e compreensão, ocasionando o enfrentamento da infecção um processo solitário. Pode-se verificar isso na fala do seguinte participante, grupo etário 23-30 anos: "Já tive vários preconceitos, principalmente por parte da família, não todos, mas alguns já me desejaram a morte”.

Parte das pessoas que vivem com HIV/AIDS desta pesquisa relataram que haviam compartilhado sua condição de HIV reagente e destacaram arrependimento de tê-lo feito. Denota-se que esses atores sociais narraram que vivenciaram afastamento de pessoas da sua rede de apoio social, consequência da sua condição de soropositividade para o HIV, pode ser observado na fala da seguinte participante, grupo etário 31-38 anos: "Sim, eu era tratada de um jeito antes e hoje sou tratada de outra maneira". Os dados apreendidos entre os participantes desta investigação são corroborados com estudos cujos autores mencionam que a soropositividade é uma doença que gera um estigma negativo que causa uma ambiguidade em torno da aceitação ou não do diagnóstico por pessoas próximas e familiares e da culpa que é depositada na pessoa (Zambenedetti \& Both, 2011).

Destaca-se outro dado relevante, como o fato de que quando se perguntou ao entrevistado se ele já havia sofrido algum preconceito ou estigma: "Sim, por ser gay e possuir AIDS, pela família e conhecidos" (sexo masculino, 47-54 anos). A doença ainda hoje é vista como algum castigo divino ou como algo que você escolheu viver de modo irregular e afrontador, fazendo com que as pessoas pensem que determinados comportamentos sexuais que fogem do padrão normativo da sociedade são determinantes para o indivíduo ser soropositivo (Seffner \& Parker, 2016).

Destarte, esta investigação apontou para uma prevalência da estigmatização social da condição de soropositivo para HIV reagente ancorada no sexo masculino, heterossexual e de classe socioeconômica baixa. Sabe-se que, diante da realidade brasileira de disparidades econômicas, sociais e cultuais, esse pode ser um indicativo de como se propaga essa enfermidade e como se dilui o preconceito entre pessoas que convivem com HIV/AIDS. Geralmente, o que produz e reproduz a discriminação/estigma social são as bases culturais, econômicas, políticas e sociais (Garbin, Garbin, Moimaz \& Carmo, 2009).

\section{Nuvem de Palavras da Estigmatização Social das Pessoas que Vivem com HIV / AIDS}

Uma diferente maneira de se dispor os dados de forma gráfica no presente córpus é a nuvem de palavras (ver Figura 2), que agrupa e organiza as palavras em função da sua frequência, sendo possível identificar as palavras-chave centrais. Nesse caso, algumas dessas palavras são: "não", "saber", "família", "preconceito", "sim" e "ninguém". 
Figura 2. Nuvem de palavras da estigmatização entre pessoas que vivem com HIV

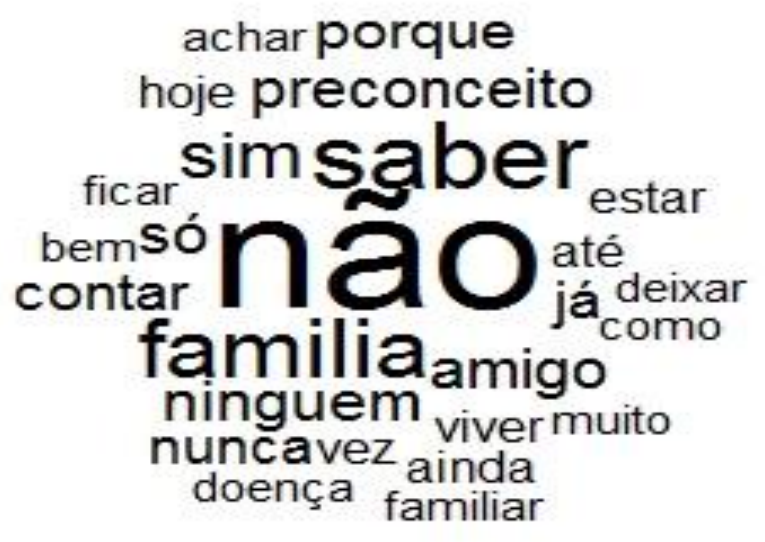

Fonte: Elaborada pelos autores.

As palavras no centro da nuvem parecem estar relacionadas aos discursos que confirmam que as pessoas não contam para pessoas próximas ou familiares que têm HIV/AIDS, por receio de sofrer alguma discriminação/estigma social. Os relatos não apresentam pontos positivos, pois até mesmo quem já contou para alguém já sofreu discriminação de alguma forma, segundo as falas dos participantes da pesquisa. Ainda, a não revelação da condição de soropositividade acarreta na omissão de vivências de discriminação e preconceitos. Tal fato é verificado em estudos prévios, os quais demonstraram que há uma associação entre a condição de soropositividade para HIV e o aumento nas vivências de estigma social e discriminação (Stringer et al., 2016; Stump, La Pergola, Cross \& Else-Quest, 2016).

A seguir é apresentado o resultado da análise de similitude, contendo as ocorrências das palavras e indicações das suas conexões.

\section{Análise de Similitude da Estigmatização Social das Pessoas que Vivem com HIV/AIDS}

A análise de similitude apresenta um desenho indicando a conexão entre as palavras, pelo qual é possível identificar as coocorrências destas (ver Figura 3). 
Figura 3. Análise de similitude da estigmatização entre pessoas que vivem com HIV

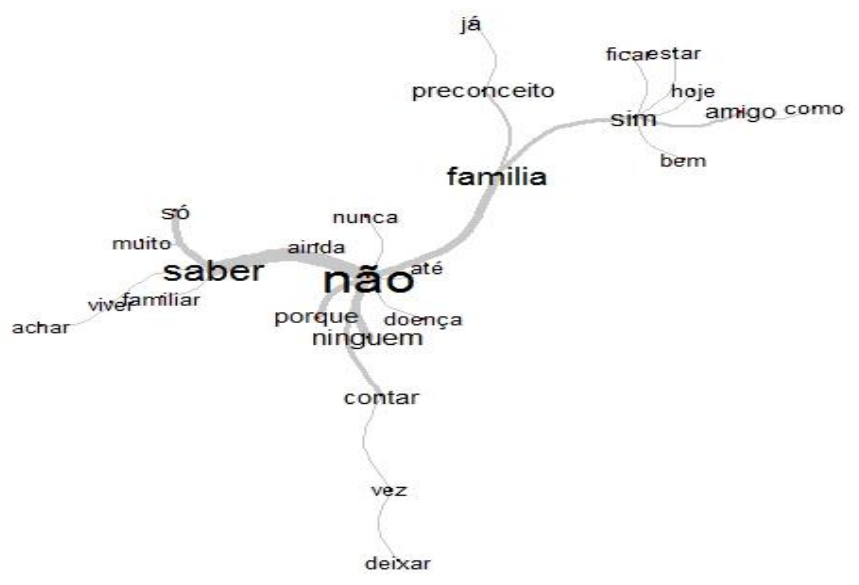

Fonte: Elaborada pelos autores.

A palavra "não" aparece no centro da árvore, dando seguimento às demais ramificações que partem dela, formando a árvore e apresentando o maior número de palavras associadas. As palavras que se seguem dão continuidade à recusa de contar ou não sobre o resultado do exame: ninguém, porque, nunca, saber. Tal fato, assim como nas análises anteriores (dendograma e nuvem de palavras), também fizeram parte das ramificações da árvore na análise de similitude, de modo que essas pessoas com HIV/AIDS, ao não revelarem a sua condição de soropositividade aos seus pares, consequentemente não vivenciaram nenhum tipo de discriminação e/ou estigma social. Em estudos anteriores, de forma consonante, a presente pesquisa demonstrou que o estigma social e a condição da soropositividade estavam associados ao sofrimento psíquico entre pessoas que vivem com HIV/AIDS (Burnham, Cruess, Kalichman, Grebler, Cherry \& Kalichman, 2016; Garett, Smith, Chiu \& Young, 2016).
O conjunto de dados desta pesquisa demonstrou, por um lado, um discurso negativo acerca da soropositividade para HIV/AIDS, tais concepções são reforçadoras de preconceitos evidenciados nas relações interpessoais; por outro lado, a construção social negativa da AIDS ainda é bem arraigada na nossa sociedade, mesmo com os avanços no tratamento e na condição de melhora de vida das pessoas com HIV reagente. Por fim, evidenciou-se entre os participantes que o não compartilhamento da condição de HIV/AIDS é uma estratégia de defesa, tendo em vista que aqueles que compartilharam sua enfermidade tiveram experiências de preconceito e/ou estigma sociais.

\section{Considerações Finais}

O presente estudo abordou a discriminação/estigma social vivenciado pelas pessoas que vivem com HIV/AIDS. Os dados apreendidos evidenciaram que o estigma social e 
o preconceito são uma realidade no contexto do HIV/AIDS. Assim, apesar de mais de três décadas passadas da epidemia e da sua mudança epidemiológica, as pessoas que convivem com HIV/AIDS internalizam estigmas. A partir dos resultados desta pesquisa, salienta-se que foi constatado que a discriminação/estigmatização social é um fator relevante a ser considerado nas políticas públicas de saúde.

Outro fator que merece destaque diz respeito ao perfil dos participantes, que eram em sua maioria de baixa renda, com nível de instrução baixo, heterossexuais e desempregados. Essas características sociodemográficas demonstram que essas pessoas estão em uma zona de maior vulnerabilidade social, sendo identificadas poucas informações e/ou métodos preventivos utilizados pelos entrevistados, que mesmo sabendo de tais riscos mantêm comportamentos sexuais de risco.

Faz-se necessário mencionar que esta pesquisa ainda revelou que a família entra em cena como uma instituição de dualidade, sendo ora acolhedora, ora discriminatória. Sabe-se que o papel da família e dos pares para o enfrentamento das adversidades no contexto do HIV/AIDS, entre eles o estigma social e o preconceito, são fatores psicossociais relevantes para atenuar a vulnerabilidade social das pessoas que vivem com HIV reagente.

Os dados deste estudo reforçam a necessidade de programas de intervenção que promovam e disseminem informação, métodos contraceptivos, entre outros. Necessitam-se, assim, de estudos que sejam mais condizentes com a população, e que tenham maior abrangência para que se tornem mais generalizados, para assim contribuírem para futuras pesquisas e disseminação de conhecimento, pois essa temática é de suma importância nos campos da saúde, educação, entre outros.

Dessa forma, espera-se que o conjunto dos dados desta pesquisa possa subsidiar futuras intervenções em saúde, com o escopo de contribuir num maior nível de resiliência, tendo em vista que este constructo poderia ser utilizado nas situações que foram descritas, pois poderia proporcionar uma qualidade de vida melhor em relação tanto da vivência do estigma e da exclusão social quanto para manejar os efeitos colaterais dos remédios, advindos dos medicamentos antirretrovirais, proporcionando uma continuidade da vida, mesmo depois da infecção pelo HIV/AIDS.

Apesar das fortalezas inerentes a esta pesquisa, é válido salientar que ela tem algumas limitações. Visto isso, sugerem-se estudos futuros com modelos explicativos que contemplem diferentes constructos psicossociais (resiliência, autoeficácia para adesão ao tratamento, estigma social, conhecimento sobre infecção pelo HIV e apoio social) de forma comparativa entre pessoas que vivem com HIV/AIDS e a população em geral.

\section{Referências}

Araújo, L. F., Teva, I., \& Bermudez, M., P. (2014). Psychological and SocioDemographic Variables Associated with Sexual Risk Behavior for Sexually Transmitted Infections/HIV. International Journal of Clinical and Health Psychology, 14, 120 127. 
Araújo, L. F., Teva, I., \& Bermudez, M. P. (2015). Resiliencia en adultos: una revisión teórica. Terapia Psicológica, 33, 257 276. Doi:10.1016/S1697-2600(14)700456.

Araújo, L. F., Teva, I., Quero, J. H., Reyes, A. O., \& Bermúdez, M. P.(2017). Analysis of Resilience and Sexual Behavior in Persons with HIV Infection. Psicologia: Reflexão e Critica, 30(1), 21-30. Doi:10.1186/s41155017-0076-6.

Associação Brasileira de Estudos Populacionais. (2012). Relatório Global do Unaids revela que 19 milhões das 35 milhões de pessoas que hoje vivem com HIV não sabem que têm os vírus. Recuperado de http://www.abep.org.br/?q=destaques/u naids-2014.

Associação Brasileira Interdisciplinar de AIDS. (2001). Estigma, discriminação e AIDS. Recuperado de http://www.abiaids.org.br/_img/media/c olecao $\% 20$ cidadania $\% 20$ direito.pdf.

Berger, B. E., Ferrans, C. E., \& Lashley F. R. (2001). Measuring Stigma in People with HIV: Psychometric Assessment of HIV Stigma Scale. Research in Nursing \& Health, 24, 518-529. Doi: 10.1002/nur.10011.

Camargo, B. V. (2005). Alceste: um programa informático de análise quantitativa de dados. In A. S. P. Moreira (Org.). Perspectivas teórico-metodológicas em representações sociais (pp. 511-539). João Pessoa: UFPB/Editora Universitária.

Camargo, B. V., Justo, A. M. (2013). Iramuteq: um software gratuito para análise de dados textuais. Temas em Psicologia, 21(2), 513-518. Doi:10.9788/TP2013.2-16

Carvalho, S. M., \& Paes, G. O. (2011). A influência da estigmatização social em pessoas vivendo com HIV/AIDS. Cad. Saúde Colet., 19(2), 157-163.

Durães, J. S. (2011). Cuidado e superação do estigma do HIV/AIDS: estágio de observação no grupo de adesão do Hospital de Clínicas da UFPR. In C. Peretti (Org.). Teologia, gênero, expressos: para onde vamos? (pp. 543-558). Curitiba, Paraná.

Galvão, A. C. (2009). Os muros (in)visiveis do preconceito: um estudo das representações sociais das pessoas que vivem com HIV/AIDS. PósGraduação em Psicologia Social, Universidade de Brasília, Brasília, Distrito Federal, Brasil.

Garbin, C. A. S., Garbin, A. J. I., Moimaz, S. A. S., \& Carmo, M. P (2009). Bioética e HIV/Aids: discriminação no atendimento aos portadores. Revista Bioética, 17(3), 511 522.

Guerra, P.P., \& Seidl, E. M. F. (2009). Crianças e adolescentes com HIV/AIDS: revisão de estudos sobre revelação do diagnóstico, adesão e estigma. Dissertação de mestrado, Universidade de Brasília, Brasília, Distrito Federal, Brasil.

Junqueira, M. F. R., Garcia-Zapata, M. T. A., Neto, S. B. C., Barbosa, H. C. F., \& Buzin, E. J. W. K. (2013). Enfrentamento de pessoas com HIV/AIDS. Rev. Enciclopédia Biosfera, 9(16), 2233.

Maldonado, M. S., Blanco, J. L. T., \& Rendón, J. C. L. (2016). Estigmatización y usos léxicos en el tratamiento informativo del $\mathrm{VIH} / \mathrm{SIDA}$ en cinco diarios mexicanos de 2012 a 2013. Nueva época, 25, 71-100.

Ministério da Saúde. (2011). Secretaria de Vigilância em Saúde: agenda estratégica. Brasília/DF.

Moreira, V., Meneses, A. M., Andrade, D. B., \& Araújo, M. C. (2010). Fenomenologia do estigma em HIV/AIDS: "coestigma". Rev. de Saúde Mental e Subjetividade da UNIPAC, 3(14), 115-131.

Nascimento, A. R. A., \& Menandro, P. R. M. (2006). Análise lexical e análise de conteúdo: uma proposta de utilização conjugada. Estudos e Pesquisas em Psicologia, 6(2), 1-17.

Programa das Nações Unidas. (2010). Global Report. Recuperado de http://www.unaids.org/globalreport/doc uments/20101123_GlobalReport_full_en .pdf. 
Programa das Nações Unidas. (2015). Estimativas sobre o HIV e AIDS no Brasil 2014.

Recuperado de http://unaids.org.br/estatisticas/.

Programa das Nações Unidas. (2015). Existe cura para o HIV?. Recuperado de http://unaids.org.br/informacoesbasicas/.

Programa das Nações Unidas. (2015). AIDS bythe numbers. Recuperado de http://www.unaids.org/sites/default/files /media_asset/AIDS_by_the_numbers_2 015_en.pdf.

Qião, S., Li, X., \& Stanton, B. (2014). Social Support and HIV-Related Risk Behaviors: A Systematic Review of the Global Literature. AIDS Behav, 18(2), 419-441. Doi:10.1007/s10461-013-0561-6.

Ratinaud, P., \& Marchand, P. (2012). Application de la méthode alceste à de "gros" corpus et stabilité des "mondes lexicaux": analyse du "Cable-Gate" avec Iramuteq. Actes des 11 eme Journées internationales d'Analyse statistique des Données Textuelles, 835-844. Doi: 10.9788/TP2013.2.

Reinert, M. (1990). Alceste: une methologie d'analyse dês donnees textualles et une application. Bulletin de Méthodologie Sociologique, 28, 24-54.

Reis, R. K., Melo, E. S., Galvão, M. T. G., \& Gir, E. (2014). Educação em saúde junto às pessoas com HIV/AIDS: proposta de intervenção interdisciplinar. Cienc Cuid Saude, 13(3), 402-410. Doi: $10.4025 / 13 i 3.21120$.

Santos, N. A. (2014). Representações sociais de mulheres que vivem com o HIV / AIDS sobre AIDS, HIV e cuidado de Enfermagem. Tese de doutorado, Universidade Federal da Bahia, Salvador, Brasil.

Secretaria de Vigilância em Saúde Ano V. (2011). Relatório de situação Ministério da saúde. Recuperado de http://bvsms.saude.gov.br/bvs/publicaco es/sistema_nacional_vigilancia_saude_pi _5ed.pdf.

Secretaria de Saúde do Piauí. (2013). Casos de
AIDS em adulto por municípios de residência do Piauí, segundo ano de notificação - 2014-2013.

Seffner, F., \& Parker, R. (2016). Desperdício da experiência e precarização da vida: momento político contemporâneo da resposta brasileira à AIDS. Comunicação Saúde Educação, 20(57), 293-304. Doi:10.1590/1807-57622015.0459.

Teva, I., Araújo, L. F., \& Bermúdez, M. P. (2018). Knowledge and Concern about STIs/HIV and Sociodemographic Variables Associated with Getting Tested for HIV among the General Population in Spain. The Journal of psychology, 1-14. Doi:10.1080/00223980.2018.1451815.

Zambenedetti, G., \& Both. N. S. (2013). "A via que facilita é a mesma que dificulta": estigma e atenção em HIV-AIDS na estratégia saúde da família - ESF Fractal. Rev. Psicol., 25(1), 41-58.

Doi:10.1590/S1984-0292201300010000.

Zambedetti, G. (2014). O paradoxo do território e os processos estigmatização da AIDS na atenção básica em saúde. Tese de doutorado, Universidade Federal do Rio Grande do Sul, Porto Alegre, Rio Grande do Sul, Brasil.
Recebido em: 9/12/2017

Aprovado em: 26/6/2018 